\title{
Liverworts, mosses and hornworts of Afghanistan - our present knowledge
}

\author{
Harald Kürschner \& Wolfgang Frey
}

Liverworts, mosses and hornworts of Afghanistan - our present knowledge. - Acta Mus. Siles. Sci. Natur., 68: 11-24, 2019.

\begin{abstract}
A new bryophyte checklist for Afghanistan is presented, including all published records since the beginning of collection activities in $1839-1840$ by W. Griffith till present. Considering several unidentified collections in various herbaria, 23 new records for Afghanistan together with the collection data can be added to the flora. Beside a new genus, Asterella, the new records include Amblystegium serpens var. serpens, Brachythecium erythrorrhizon, Bryum dichotomum, B. elwendicum, B. pallens, B. weigelii, Dichodontium palustre, Didymodon luridus, D. tectorum, Distichium inclinatum, Entosthodon muhlenbergii, Hygroamblystegium fluviatile subsp. fluviatile, Oncophorus virens, Orthotrichum rupestre var. sturmii, Pogonatum urnigerum, Pseudocrossidium revolutum, Pterygoneurum ovatum, Schistidium rivulare, Syntrichia handelii, Tortella inflexa, T. tortuosa, and Tortula muralis subsp. obtusifolia. Therewith the number of species increase to 24 liverworts, 246 mosses and one hornwort. In addition, a historical overview of the country's exploration and a full biogeography of Afghan bryophytes is given.
\end{abstract}

Key words: Bryophytes, checklist, flora, phytodiversity.

\section{Introduction}

Recording, documentation, identification and classification of organisms is a primary tool and essential step in plant sciences and ecology to obtain detailed knowledge on the flora of a country. In many countries, such as Afghanistan, however, our knowledge on plant diversity, function, interactions of species and number of species in ecosystems is very limited and far from being complete. Especially bryophytes (liverworts, mosses, hornworts) are often widely neglected and only seldom in the focus of scientific interest and research (e.g., Frey \& Kürschner 2009).

Although large areas of Afghanistan consist of high mountain regions, bryophytes especially mosses, may form extensive cover, especially on rocks in mountainous regions, in flushes, near watercourses and springs, and in sites where snow-melt moisture is available for an extended part of the growing season. There they often dominate the vegetation cover and control the vegetation pattern and dynamics of associated flowering plants. Even in the semihumid or semi-arid forests and woodland of NE- and SW-Afghanistan and in the arid and semiarid steppes and deserts of W- and S-Afghanistan, bryophytes are widely distributed on marly or clayey soils and are an important part of the often sparsely vegetated microphytic crusts. Especially Pottiaceae, the 'mosses of harsh environments' (Zander 1993) play an essential role in soil and crust stabilization of deserts, hindering soil erosion.

As bryophytes are perfect organisms to serve as indicators for ecological disturbances and air pollution (Frey 1989, Frahm 1998, Frahm et al. 2008) the knowledge of species is of fundamental importance for understanding ecosystem development (succession stages), phytodiversity and nature conservation, especially with respect to the increasing human impact and global warming effects.

In the following a new species catalogue for Afghanistan is presented to give inside in the bryophyte diversity of the country. It is based on all published records since the beginning of bryophyte research in Afghanistan (Griffith 1849), various till present unidentified collect- 
ions and the 'Conspectus Bryophytorum Orientalum et Arabicorum' (Frey \& Kürschner 1991).

The new checklist responds to the tools of the Conservation on Biological Diversity and the Targe 1 of the updated Global Strategy for Plant Conservation to achieve a list of all known plants. It is a further step to integrate Afghanistan into the Global Network of floristic knowledge and provide easy access to taxonomic information.

\section{Bryological exploration and collectors (following Frey \& Kürschner 2009)}

The bryological exploration of Afghanistan dates back to 1839-1840, when W. Griffith collected and reported 80 taxa from the area, unfortunately without locality data (Griffith 1849). He was followed by Surgeon-Major J.E.T. Aitchison in 1879 and 1880, who collected plants in the Kuram Valley during the $2^{\text {nd }}$ Anglo-Afghan war, adding two taxa to the country's flora (Aitchison 1881-1882). A third taxon collected by Aitchison in Afghanistan 1884-1885 is Pterygoneurum media, described as new to science by Salmon (1899; basionym Tortula media, sine loco, herb K, no. 183).

After those early collections in the $19^{\text {th }}$ century, no others are known till in $1938 \mathrm{Th}$. Herzog published the bryological result of the 'Deutsche Hindukusch-Expedition 1935'. He recorded two liverworts and 31 mosses (Herzog 1938).

From 1949 to 1951, intensive phytosociological field studies on the high mountain vegetation of central, east and north Afghanistan were carried out by the Austrian botanist A. Gilli. He also collected bryophytes, which were identified by J. Fröhlich at Vienna (Fröhlich 1955). Within this collection, six taxa were described as new to science, some of them known at present only from the type locality.

In 1955, S. Kitamura visited Afghanistan as a member of the 'Kyoto University Scientific Expedition'. His collections include three liverworts and 27 mosses from Nuristan, published by Kitagawa (1964). With the beginning of the 60s and 70s of the last century, there was an increasing interest on the flora and vegetation of this fascinating country. Several botanists took the opportunity to visit Afghanistan and collect plants within the framework of various research projects. This period of intensive exploration starts with the field trips by C. v. Regel in 1958 and 1960 (results presented by Bizot 1962), followed by I. C. Hedge and P. Wendelbo (AprilJuly) in 1962 (21 taxa published by Henderson \& Prentice (1963), and K. H. Rechinger in MayAugust 1962. Rechinger's collection again was identified by J. Fröhlich who described 10 taxa as new to science (Fröhlich 1964). Altogether, Fröhlich described 14 new species and two new varieties from Afghanistan - six of them, however, are sunk in synonymy and three remain doubtful. The other five taxa are known only from the type localities and mostly refer to critical or somewhat problematical taxa of uncertain taxonomic position.

Next to be mentioned are Admiral Paul and Mrs Furse (April-May 1964, cf. Townsend 1966), followed by W. Frey 1965 (cf. Frey 1969). Further collections during this period came from S.-W. Breckle 1968-1969 (herb. S.-W. Breckle), D. Dieterle 1969, 1970 (herb. M), E. Koblmüller 1968 (herb. M), I. Kukkonen 1972 (herb. H), D. Podlech 1965-1971 (herb. M), M. Steiner 1970 (herb. M. Steiner, M), W. Frey 1974 and P. Uotila 1972 (herb. H). Together with the older collections of H. O. Volk (1950, 1952; dupl. herb. W. Frey), the results partly were published in Frey $(1972,1974)$ and Frey \& Kürschner $(1991,2009)$. At the end of the 60s, M. Bizot and G. Kilbertus travelled and collect in the country (Kilbertus \& Bizot 1971).

As far as we know, the last bryophyte collections from the country comes from H. Huss 1975, a member of the Austrian 'Exploration Pamir ' 75 - Österreichisches Forschungsunternehmen' (herb. GZU), who collected in the Wakhan corridor (Darrah-e Issik, Koh-e Pamir; cf. Frey \& Kürschner 2009) and V. Plášek (Ostrava) from the Tajik-Afghan border (June 2011, cf. Plášek et al. 2014). 


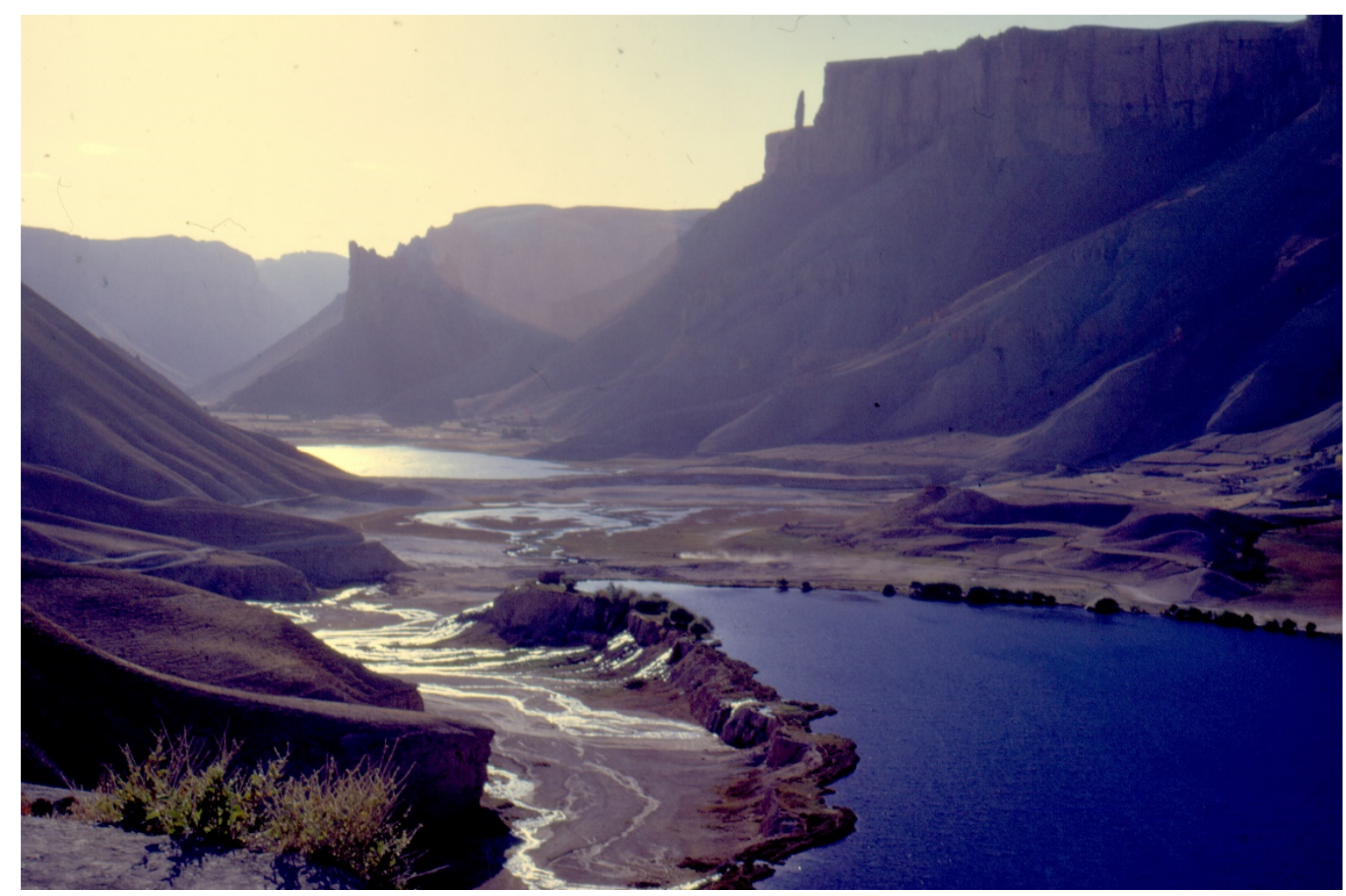

Fig 1: Landscape of Central Afghanistan. Valley near Band-e Amir.

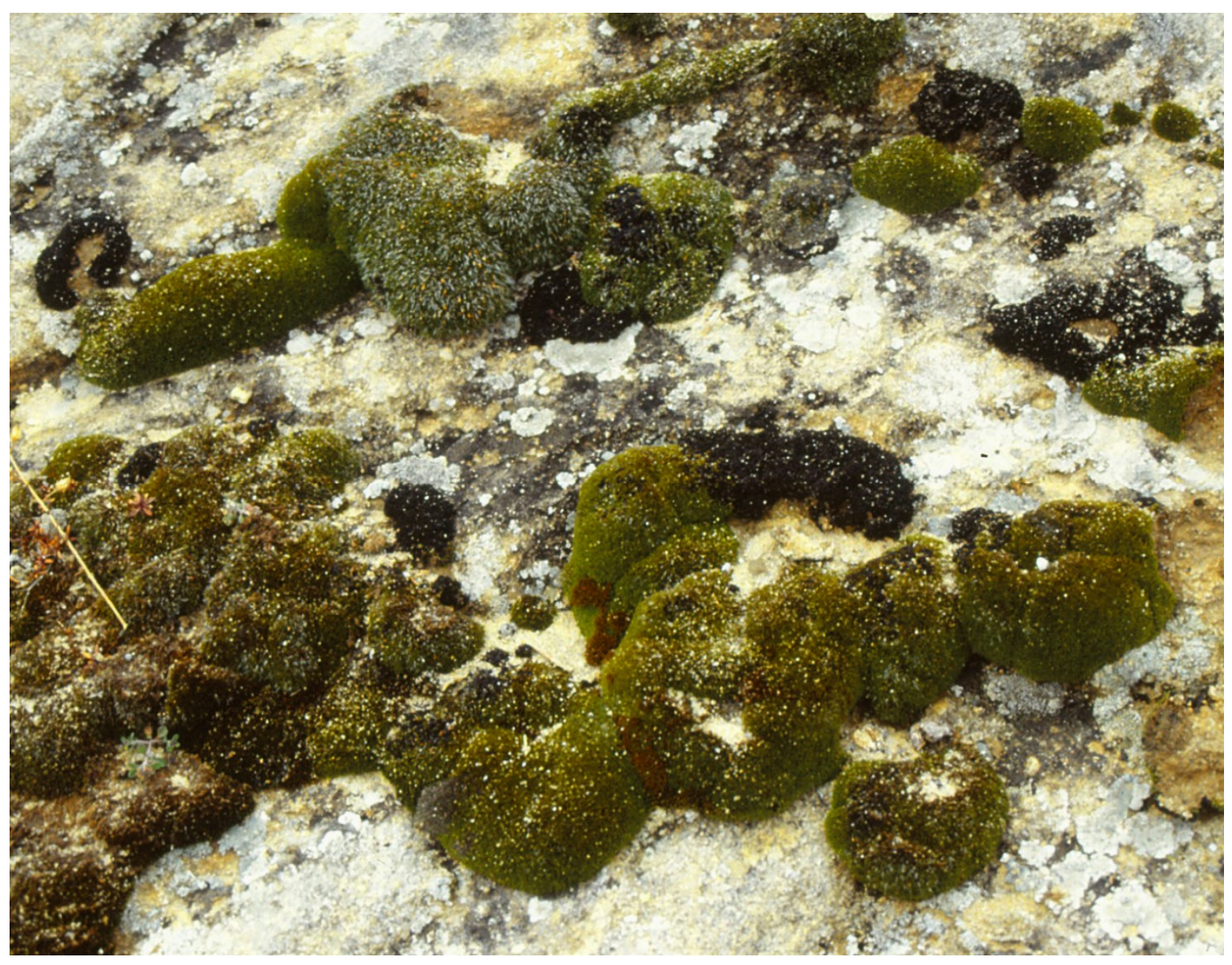

Fig 2: Typical bryophyte rock community with various acrocarpous mosses from the Grimmiaceae family. 
At present, in cooperation with the United Nations Environment in Afghanistan and the National Environmental Protection Agency a field guide and textbook of bryophytes of Afghanistan is in press, which is useful for educational purposes and the re-established Afghan institutions in Kabul (Kürschner et al. 2019).

\section{Taxa described from Afghan plant material}

Based on plant material collected in Afghanistan, 19 taxa have been described as species new to science. Especially J. Fröhlich (Vienna) recorded many new species based on material collected by K. H. Rechinger in 1962. Most of them are known only from a single collection, represented by the type specimen (holotype) and kept at Vienna (herb. W). With increasing knowledge on the taxonomy and distribution of species, however, quite a number of these former 'endemics' have sunken into synonymy and turned out to be conspecific with more widely distributed and/or common species in South-West Asia.

For historical and taxonomical reasons, we present an overview of the taxa which have been described from Afghan plant material. At present accepted names are given both, in bold and italic.

Anoectangium pleuroweisioides J.Fröhl. = Molendoa hornschuchiana (Hook.) Lindb. ex Limpr.

Barbula cucullata J.Fröhl. = Didymodon nicholsonii Culm.

Brachythecium plumosum (Hedw.) Schimp. var. laxoalare Herzog

Known only from the type. If this taxon merits taxonomic recognition, a new combination under Sciuro-hypnum plumosum (Hedw.) Ignatov \& Huttunen is required.

Bryum funckioides J.Fröhl.

Known only from the type.

Bryum purpureo-lucidum J.Fröhl.

Known only from the type.

Dicranoweisia brevifolia Dixon \& Herzog

Known only from the type.

Didymodon recurvirostris (Hedw.) Jenn. var. obtusifolius J.Fröhl.

The identity of this name has not been reviewed. If it merits recognition, a new combination under Bryoerythrophyllum recurvirostrum (Hedw.) P.C.Chen may be required. However, in contrast, Kürschner (2007) stated that it may be conspecific with B. rubrum (Jur. ex Geh.) P.C.Chen

Grimmia cardotii var. gymnostoma J.Fröhl.

Known only from the type. If this taxon deserves taxonomic recognition, a new combination under G. poecilostoma Cardot \& Sebille is required.

Hydrogonium heterophyllum J.Fröhl. = Splachnobryum aquaticum Müll.Hal.

Hydrogonium patulifolium J.Fröhl.

Hygrohypnum smithii (Sw.) Broth. var. denticulata Kilbert.

Known only from the type.

Hymenostylium diversifolium J.Fröhl. = Hymenostylium recurvirostrum (Hedw.) Dixon

Mniobryum tortifolium J.Fröhl. = Pohlia tortifolia (J.Fröhl.) W.Frey \& Kürschner

Tortula cabulica J.Fröhl. = Didymodon australasiae (Hook. \& Grev.) R.H.Zander

Tortula cucullifolia J.Fröhl. = Didymodon australasiae (Hook. \& Grev.) R.H.Zander

Tortula pseudodesertorum J.Fröhl. = Syntrichia caninervis var. caninervis Mitt.

Tortula pseudohandelii J.Fröhl. = Syntrichia caninervis var. pseudodesertorum (Vondr.) M.T.Gallego

Tortula subtranscaspica J.Fröhl.

Weissia viridula var. remotidens Herzog

If this variety deserves taxonomic recognition, a new combination under Weissia controversa Hedw. is necessary.

\section{Checklist of Afghan bryophytes}

Within bryophyte taxonomy, a strong segregation can be observed during the last years, where recent molecular data lead to a splitting of many 'traditional, old groups' and families (Kürschner \& Frey 2011). To a great extent, the treatment of families and genera in the following checklist strictly follows these studies and the most recent tentative system proposed by Frey \& Stech (2009). 
The sequence of families, genera and species is strictly alphabetical to make using the checklist easier.

Nomenclature is in accordance with Ros et al. (2007) in liverworts and Ros et al. (2013) in mosses, except for extra-European taxa or when these lists differ greatly from modern concepts. In these cases, TROPICOS (www.tropicos.org) was consulted. The following list include all published records from Afghanistan since the beginning of collection activities (Griffith 1849) till present. The references given in brackets refer to the literature where the records were published for the first time. Altogether, 271 taxa are listed (24 liverworts, 246 mosses and one hornwort).

In addition, 23 new records for Afghanistan were presented, together with the collection data and collectors.

\section{MARCHANTIOPHYTA}

\section{Aneuraceae}

Riccardia palmata (Hedw.) Carruth. (Frey \& Kürschner 2009)

\section{Aytoniaceae}

Asterella spec. (Genus new to Afghanistan); KABUL: $10 \mathrm{~km} \mathrm{~W}$ Sarobi, 3435'N 69³8'E, $1200 \mathrm{~m}$, Kalkfelsspalten, 18. April 1969 S.-W.Breckle

Mannia androgyna (L.) A.Evans (Fröhlich 1955)

M. fragrans (Balb.) Frye \& L.Clark (Frey 1974)

Plagiochasma appendiculatum Lehm. \& Lindenb. (Bischler 1978)

P. cordatum Lehm. \& Lindenb. (Bischler 1979)

Reboulia hemisphaerica (L.) Raddi (Kitagawa 1964)

\section{Cleveaceae}

Athalamia hyalina (Sommerf.) S.Hatt. (Frey 1974)

A. spathysii (Lindenb.) S.Hatt. (Frey 1974)

\section{Conocephalaceae}

Conocephalum conicum (L.) Dumort. (Fröhlich 1955)

\section{Lophocoleaceae}

Chiloscyphus polyanthos (L.) Corda (Kitagawa 1964)

\section{Marchantiaceae}

Marchantia paleacea Bert. (Bischler-Causse 1989)

M. papillata subsp. grossibarba (Steph.) Bischl. (Bischler-Causse 1989)

M. polymorpha subsp. polymorpha (Griffith 1849)

M. quadrata Scop. (Fröhlich 1955) [Syn. Preissia quadrata (Scop.) Nees]

Pelliaceae

Pellia endiviifolia (Dicks.) Dumort. (Fröhlich 1955)

\section{Porellaceae}

Porella cordaeana (Hübener) Moore (Müller 1954-1957, p. 1219, in Frey 1974)

P. platyphylla (L.) Pfeiff. [Herzog 1939 as P. decurrens (Steph.) Hattori, cf. Hattori 1969, 1978]

P. trigonifolia (Steph.) Paris (Herzog 1938). The record is doubtful and probably refers to P. platyphylloidea (Schweinf.) Lindb.

\section{Radulaceae}

Radula complanata (L.) Dumort. (Müller 1954-1957, p. 1192, in Frey 1974)

\section{Ricciaceae}

Riccia bifurca Hoffm. (Kürschner 1996)

R. fluitans L. (Fröhlich 1955)

\section{Targioniaceae}

Targionia hypophylla L. (Frey 1974)

\section{Wiesnerellaceae}

Wiesnerella denudata (Mitt.) Steph. (Kitagawa 1964) 


\section{BRYOPHYTA}

\section{Amblystegiaceae}

Amblystegium serpens (Hedw.) Schimp. var. juratzkanum (Schimp.) Rau \& Herv. (Fröhlich 1964)

A. serpens (Hedw.) Schimp. var. serpens (Hedw.) Schimp. (new to Afghanistan); KAPISA: Tagab-Alisang, way to Kotal Spe, 7 September 1974 W. Frey 74-80

Callialaria curvicaulis (Jur.) Ochyra [Gilli 1971, as Cratoneuron curvicaule (Jur.) G.Roth]

Campylium stellatum (Hedw.) C.E.O.Jensen (Fröhlich 1955)

Cratoneuron filicinum (Hedw.) Spruce (Fröhlich 1955)

Drepanocladus aduncus (Hedw.) Warnst. (Herzog 1938)

D. turgescens (T.Jensen) Broth. (Frey 1972)

Hamatocaulis vernicosus (Mitt.) Hedenäs (Kilbertus \& Bizot 1971)

Hygroamblystegium fluviatile (Hedw.) Loeske subsp. fluviatile (new to Afghanistan); BADAKSHAN: Darrah-i Jokham, Hazarat-i Sayet, 2700 m, Bewässerungskanal, 3. August 1974 W. Frey M27; NURISTAN: Bashgal Quelltäler, unteres Darrah-i Pushal, 3300 m, in einem Bach, 5. September D.Podlech (M)

H. fluviatile (Hedw.) Loeske subsp. noterophilum (Sull. \& Loeske) Grout (Fröhlich 1964)

H. humile (P.Beauv.) Vanderp., Goffinet \& Hedenäs [Fröhlich 1964, incl. Leptodictyum kochii (Schimp.) Warnst. var. curvipes (Schimp.) Warnst.]

H. tenax (Hedw.) Jenn. (Kürschner \& Frey 2006)

H. varium (Hedw.) Mönk. (Fröhlich 1955)

Hygrohypnum luridum (Hedw.) Jenn. var. subsphaericarpon (Brid.) C.O.E.Jensen (Herzog 1938)

H. smithii (Sw.) Broth. var. denticulata Kilbertus (Kilbertus \& Bizot 1971, holotype)

Leptodictyum riparium (Hedw.) Warnst. (Fröhlich 1955)

Palustriella commutata (Hedw.) Ochyra (Herzog 1938)

P. decipiens (De Not.) Ochyra (Frey \& Kürschner 2009)

P. falcata (Brid.) Hedenäs (Fröhlich 1955)

Sanionia uncinata (Hedw.) Loeske (Herzog 1938)

Amphidiaceae

Amphidium lapponicum (Hedw.) Schimp. (Kitagawa 1964)

Aongstroemiaceae

Dichodontium palustre (Dicks.) M.Stech (new to Afghanistan); HeRAT: Ziarat-i Mullah-i Khwaja-i Koh, 20$30 \mathrm{~km} \mathrm{NW}$ Herat, c. $34,5^{\circ} \mathrm{N} 61,9^{\circ} \mathrm{E}, 1180 \mathrm{~m}$, Wasserfall, 14. März 1969 S.-W.Breckle

\section{Aulacomniaceae}

Aulacomnium palustre (Hedw.) Schwägr. (Herzog 1938)

\section{Bartramiaceae Schwägr.}

Bartramia aprica Müll.Hal. (Kitagawa 1964, as B. stricta Brid.)

Philonotis caespitosa Jur. (Fröhlich 1955)

P. calcarea (Bruch \& Schimp.) Schimp. (Fröhlich 1955)

P. falcata (Hook.) Mitt. (Fröhlich 1955)

P. fontana (Hedw.) Brid. (Aitchison 1881-1882)

P. marchica (Hedw.) Brid. (Herzog 1938)

P. seriata Mitt. (Frey 1969)

P. thwaitesii Mitt. (Kitagawa 1964)

P. turneriana (Schwägr.) Mitt. (Fröhlich 1955)

\section{Brachytheciaceae}

Brachytheciastrum collinum (Schleich. ex Müll.Hal.) Ignatov \& Huttunen (Frey 1972)

B. olympicum (Jur.) Vanderp., Goffinet \& Hedenäs (Fröhlich 1955)

B. trachypodium (Brid.) Ignatov \& Huttunen (Henderson \& Prentice 1963)

B. velutinum (Hedw.) Ignatov \& Huttunen (Fröhlich 1955)

Brachythecium campestre (Müll.Hal.) Schimp. (Fröhlich 1964)

B. erythrorrhizon Schimp. (new to Afghanistan); KABUL: Paghman, Begtut, 2500 m, 21. August 1951 O.H.Volk

s.n.

B. microsericeum Dixon (Herzog 1938)

B. mildeanum (Schimp.) Schimp. (Frey \& Kürschner 2009)

B. myurelliforme Dixon (Herzog 1938)

B. rivulare Schimp. (Kitagawa 1964)

B. rutabulum (Hedw.) Schimp. (Henderson \& Prentice 1963) [Incl. B. rutabulum var. turgescens Limpr.]

B. salebrosum (Hoffm. ex F.Weber \& D.Mohr) Schimp. var. sericeum (Fröhlich 1964)

Eurhynchium striatum (Hedw.) Schimp. (Kürschner \& Frey 2006) 
Eurhynchiastrum pulchellum (Hedw.) Ignatov \& Huttunen var. diversifolium (Schimp.) Ochyra \& Żarnowiec (Fröhlich 1955)

Homalothecium sericeum (Hedw.) Schimp. (Kitagawa 1964)

Oxyrrhynchium hians (Hedw.) Loeske (Frey \& Kürschner 2009)

Platyhypnidium riparioides (Hedw.) Cardot (Fröhlich 1955)

Rhynchostegiella tenella (Dicks.) Limpr. (Frey \& Kürschner 2009)

Rhynchostegium confertum (Dicks.) Schimp. (Frey \& Kürschner 2009)

$R$. murale (Hedw.) Schimp. (Fröhlich 1964)

$R$. patulifolium Cardot \& Thér. (Fröhlich 1964)

Sciuro-hypnum plumosum (Hedw.) Ignatov \& Huttunen (Herzog 1938) [Incl. Brachythecium plumosum

(Hedw.) Schimp. var. laxoalare Herzog (Herzog 1938, holotype]

S. populeum (Hedw.) Ignatov \& Huttonen (Kilbertus \& Bizot 1971)

Scorpiurium deflexifolium (Solms) M.Fleisch. \& Loeske (Kilbertus \& Bizot 1971)

\section{Bryaceae Schwägr.}

Brachymenium exile (Dozy \& Molk.) Bosch \& Sande Lac. (Kitagawa 1964) [Syn. Gemmabryum exile (Dozy \& Molk.) J.R.Spence \& H.P.Ramsay]

Bryum Hedw.

Note: Bryum is a large and exceedingly difficult genus and treated here in a traditional way. Based on molecular data it is split into five genera: Bryum Hedw. s.str., Gemmabryum J.R.Spence \& H.P.Ramsay, Imbribryum N.Pedersen, Ptychostomum Hornsch. and Rosulabryum J.R.Spence.

Bryum algovicum Sendtn. ex Müll.Hal. (Kilbertus \& Bizot 1971) [Syn. Ptychostomum compactum Hornsch.]

B. alpinum Huds. ex With. (Fröhlich 1955, as B. alpinum var. viride Husn.) [Syn. Imbribryum alpinum (Huds. ex With.) N.Pedersen]

B. archangelicum Bruch \& Schimp. [Henderson \& Prentice 1963, as B. inclinatum (Brid.) Turton] [Syn. Ptychostomum archangelicum (Bruch \& Schimp.) J.R.Spence]

B. argenteum Hedw. (Fröhlich 1955)

B. caespiticium Hedw. (Fröhlich 1955) [Syn. Ptychostomum imbricatulum (Müll.Hal.) D.T.Holyoak \& N.Pedersen]

B. capillare Hedw. (Fröhlich 1955) [Syn. Rosulabryum capillare (Hedw.) J.R.Spence]

B. cellulare Hook. in Schwägr. (Fröhlich 1964)

B. creberrimum Taylor (Fröhlich 1955) [Syn. Ptychostomum creberrimum (Taylor) J.R.Spence \& H.P.Ramsay]

B. dichotomum Hedw. (new to Afghanistan) [Syn. Gemmabryum dichotomum (Hedw.) J.R.Spence \& H.P.Ramsay]; BADAKSHAN: Wakhan, Großer Pamir, Issiktal, 4900 m, zwischen Blockwerk, 3. August 1975 H.Huss 16 (GZU), Exploration Pamir 1975; Helmand: 90 km N Girishk, $35 \mathrm{~km} \mathrm{~N}$ Musa Qala, 3248’N 6450’E, 1200 m, feuchter Carex stenophylla Rasen, 18. März 1969 S.-W.Breckle

B. elegans Nees (Fröhlich 1964) [Syn. Rosulabryum elegans (Nees) Ochyra]

B. elwendicum C.Fehlner (new to Afghanistan); BADAKSHAN: Darrah-i Shahran, 3500 m, feuchte Stelle, 18. August 1974 W.Frey 74-63; GHAZNI: Dasht-i Nawor, S-Umrahmung, 33²2’E 6750’E, 4100 m, VulkanitTuffe, Blockhalde, 04. Juni 1969 S.-W.Breckle s.n.

B. funckioides J.Fröhl. (Fröhlich 1955, holotype)

B. funkii Schwägr. (Frey \& Kürschner 2009) [Syn. Gemmabryum funkii (Schwägr.) J.R.Spence]

B. kunzei Hornsch. (Fröhlich 1955) [Syn. Ptychostomum kunzei (Hornsch.) J.R.Spence]

B. lanatum (P.Beauv.) Brid. (Fröhlich 1955)

B. neodamense Itzigs. (Kilbertus \& Bizot 1971) [Syn. Ptychostomum neodamense (Itzigs.) J.R.Spence]

B. pallens Sw. ex anon. (new to Afganistan) [Syn. Ptychostomum pallens (Sw. ex anon.) J.R.Spence]; BADAKSHAN: Darrah-i Parshui, 4100 m, Flußufer, auf Urgestein, 27. Juli 1965 W.Frey; BAGHLAN: Salang pass, $2 \mathrm{~km}$ N of Salang tunnel, $3000 \mathrm{~m}$, in small brook running from snow lay, 23. July 1972 P.Uotila 18791 (H)

B. pallescens Schwägr. (Fröhlich 1955) [Syn. Ptychostomum pallescens (Schwägr.) J.R.Spence]

B. pseudotriquetrum (Hedw.) P.Gaertn., E.May. \& Scherb. var. bimum (Schreb.) D.T.Holyoak \& N.Pedersen (Fröhlich 1955) [Syn. Ptychostomum bimum (Schreb.) J.R.Spence]

B. pseudotriquetrum (Hedw.) P.Gaertn., E.May. \& Scherb. var. pseudotriquetrum (Fröhlich 1964) [Syn. Ptychostomum pseudotriquetrum (Hedw.) J.R.Spence \& H.P.Ramsay ex D.T.Holyoak \& N.Pedersen]

B. purpureo-lucidum J.Fröhl. (Fröhlich 1955, holotype)

B. recurvulum Mitt. (Kürschner 1996)

B. schleicheri Schwägr. (Fröhlich 1955) [Syn. Ptychostomum schleicheri (Schwägr.) J.R.Spence]

B. syriacum Lorentz var. humile Schiffn. (Fröhlich 1964)

B. syriacum Lorentz var. syriacum (Fröhlich 1955)

B. torquescens Bruch \& Schimp. (Fröhlich 1964) [Syn. Rosulabryum torquescens (Bruch \& Schimp.) J.R.Spence] 
B. turbinatum (Hedw.) Turner (Fröhlich 1955, as B. syriacum) [Syn. Ptychostomum turbinatum (Hedw.) J.R.Spence]

B. weigelii Spreng. (new to Afghanistan) [Syn. Ptychostomum weigelii Spreng.) J.R.Spence]; BADAKSHAN: Darrah-i Parshui, 3700 m, feuchter Granit, 14. Juli 1965 W. Frey 1812

Plagiobryum demissum (Hook.) Lindb. (Kitagawa 1964) [Syn. Ptychostomum demissum (Hook.) Holyoak \& N.Pedersen]

\section{Dicranellaceae}

Dicranella howei Renauld \& Cardot [Fröhlich 1964, as D. varia (Hedw.) Schimp.) var. tenuifolia (Bruch) Bruch \& Schimp.; cf. Crundwell \& Nyholm 1977]

D. varia (Hedw.) Schimp. (Kilbertus \& Bizot 1971)

\section{Ditrichaceae}

Ceratodon purpureus (Hedw.) Brid. (Fröhlich 1955)

Distichium capillaceum (Hedw.) Bruch \& Schimp. var. capillaceum (Fröhlich 1955)

D. capillaceum (Hedw.) Bruch \& Schimp. var. compactum (Hueb.) Torre \& Sarnth. (Fröhlich 1955)

D. inclinatum (Hedw.) Bruch \& Schimp. (new to Afghanistan); BADAKsHAN: Wakhan, Wazitpass-Seitental Sabazkatsch, 3702N, 7254'E, 4900 m, 04.08.1968 S.-W.Breckle; BADAKSHAN: Darrah-i Shahran, 4200 m, Felsspalten, 18. August 1974 W. Frey 74-66; BADAKSHAN: Darrah-i Jokham, 4400 m, Steinschutt, 4. August 1974 W. Frey 74-81

Ditrichum flexicaule (Schwägr.) Hampe (Kilbertus \& Bizot 1971)

D. pusillum (Hedw.) Hampe (Bizot 1962)

\section{Drummondiaceae}

Drummondia sinensis Müll.Hal. [Fröhlich 1955, misnamed as D. prorepens (Hedw.) E.Britton]

D. thomsonii Mitt. (Herzog 1938)

\section{Encalyptaceae}

Encalypta alpina $\mathrm{Sm}$. (Kürschner 1996)

E. intermedia Jur. (Fröhlich 1955)

E. rhaptocarpa Schwägr. (Townsend 1966)

E. vulgaris Hedw. (Herzog 1938)

Fabroniaceae Schimp.

Fabronia pusilla Raddi (Fröhlich 1955)

\section{Fissidentaceae}

Fissidens diversifolius Mitt. (Fröhlich 1955)

F. grandifrons Brid. (Herzog 1938)

F. schmidii Müll.Hal. (Herzog 1938)

F. viridulus (Sw. ex anon.) Wahlenb. (Kürschner 1996)

\section{Fontinalaceae}

Fontinalis antipyretica Hedw. (Kilbertus \& Bizot 1971)

F. hypnoides C.Hartm. var. duriaei (Schimp.) Husn. (Kitagawa 1964)

\section{Funariaceae}

Entosthodon angustifolius Jur. \& Milde (Fröhlich 1964)

E. attenuatus (Dicks.) Bryhn (Fröhlich 1955)

E. buseanus Dozy \& Molk. (Fröhlich 1955, as Funaria wallichii Mitt.)

E. handelii (Schiffn.) Laz. (Fröhlich 1955)

E. muhlenbergii (Turner) Fife (new to Afghanistan); BADAKSHAN: Kokca-Gebiet, Darrah-i Jokham, 2500 m, Bachrand, 31. Juli 1974 W. Frey 74-29a

Funaria brotherusii J.Fröhl. (Herzog 1938)

F. hygrometrica Hedw. (Fröhlich 1955)

F. microstoma Bruch ex Schimp. (Kürschner 1996)

Physcomitrium pyriforme (Hedw.) Bruch \& Schimp. (Fröhlich 1955)

\section{Grimmiaceae}

Coscinodon cribrosus (Hedw.) Spruce (Henderson \& Prentice 1963)

Grimmia alpestris (F.Weber \& D.Mohr) Schleich. (Muñoz 1998)

G. anodon Bruch \& Schimp. (Fröhlich 1964)

G. capillata De Not. [Fröhlich 1964, as G. mesopotamica Schiffn.; Greven 1995, as G. crinita Brid. var. capillata (De Not.) De Not.]

G. cardotii Hérib. var. gymnostoma J.Fröhl. (Fröhlich 1964, holotype) 
G. crassifolia Broth. var. crassifolia Li (Fröhlich 1964)

A taxon with a somewhat confusing synonymy. Lectotypified and synonymized with G. poecilostoma Cardot \& Sebille ex Limpr. by Muñoz (1999), respectively G. tergestina Bruch \& Schimp. (Maier 2010), both synonyms of G. crinitoleucophaea Cardot (cf. Greven 2003)

G. crassifolia Broth. var. cucullata Baumgartner (Greven 1995)

According to Muñoz \& Pando (2000) a synonym G. tergestina Bruch \& Schimp. = G. crinitoleucophaea Cardot. The identity of both varieties, however is uncertain (cf. Ros et al. 2013)

G. crinita Brid. (Kitagawa1964)

G. crinitoleucophaea Cardot (Fröhlich 1964)

G. donniana Sm. (Kilbertus \& Bizot 1971)

G. laevigata (Brid.) Brid. (Fröhlich 1955)

G. montana Bruch \& Schimp. (Henderson \& Prentice 1963)

G. orbicularis Bruch ex Wilson (Fröhlich 1964, as var. persica)

G. ovalis (Hedw.) Lindb. (Herzog 1938)

G. plagiopoda Hedw. (Kilbertus \& Bizot 1971)

G. pulvinata (Hedw.) Sm. (Fröhlich 1955)

G. sessitiana De Not. (Muñoz 1998)

G. tergestina Tomm. ex Bruch \& Schimp. (Fröhlich 1955)

G. trichophylla Grev. (Fröhlich 1964)

Schistidium apocarpum (Hedw.) Bruch \& Schimp. (Fröhlich 1964; cf. Blom 1996)

S. flaccidum (De Not.) Ochyra (Fröhlich 1955)

S. platyphyllum (Mitt.) H.Perss. [Fröhlich 1964, as S. alpicola auct. non (Hedw.) Limpr. var. latifolia (J.E.Zetterst.) H.A.Crum \& L.E.Anderson]

S. rivulare (Brid.) Podp. (new to Afghanistan); HelmAND: Shersad bei Baghran, 2000 m, feuchte schattige Kalkfelsen, 16. März 1969 S.-W.Breckle

\section{Hedwigiaceae}

Hedwigia ciliata (Hedw.) P.Beauv. (Herzog 1938)

\section{Hypnaceae}

Calliergonella cuspidata (Hedw.) Loeske (Frey \& Kürschner 2009)

Hypnum cupressiforme Hedw. (Herzog 1938)

H. revolutum (Mitt.) Lindb. (Frey 1972)

H. vaucheri Lesq. (Ando 1976)

Taxiphyllum maniae (Renauld \& Paris) M.Fleisch. (Herzog 1938)

\section{Leskeaceae}

Haplocladium angustifolium (Hampe \& Müll.Hal.) Broth. (Kitagawa 1964)

Leskea polycarpa Hedw. (Frey \& Kürschner 2009)

Pseudoleskea incurvata (Hedw.) Loeske (Fröhlich 1955)

Pseudoleskeella tectorum (Funck ex Brid.) Kindb. ex Broth. (Fröhlich 1955)

\section{Leucodontaceae}

Leucodon sciuroides (Hedw.) Schwägr. (Herzog 1938)

\section{Meesiaceae}

Leptobryum pyriforme (Hedw.) Wilson (Herzog 1938)

\section{Mniaceae}

Mnium heterophyllum (Hook.) Schwägr. (Herzog 1938)

M. marginatum (Dicks.) P.Beauv. (Herzog 1938)

Pohlia andalusica (Höhm.) Broth. (Frey \& Kürschner 2009)

P. camptotrachela (Renauld \& Cardot) Broth. (Frey \& Kürschner 2009)

P. cruda (Hedw.) Lindb. (Fröhlich 1955)

P. obtusifolia (Vill. ex Brid.) L.F.Koch (Fröhlich 1955)

P. tortifolia (J.Fröhl.) W.Frey \& Kürschner [Fröhlich 1964, as Mniobryum tortifolium J.Fröhl. (holotype)]

P. wahlenbergii (F.Weber \& D.Mohr) A.L.Andrews var. glacialis (Brid.) E.F.Warb. (Fröhlich 1964)

P. wahlenbergii (F.Weber \& D.Mohr) A.L.Andrews var. wahlenbergii (Fröhlich 1955)

\section{Orthotrichaceae}

Nyholmiella gymnostoma (Bruch ex Brid.) Holmen \& Warncke (Fröhlich 1955)

N. obtusifolia (Brid.) Holmen \& Warncke (Fröhlich 1955)

Orthotrichum anomalum Hedw. (Herzog 1938)

O. crenulatum Mitt. (Lewinsky 1992)

O. cupulatum Hoffm. ex Brid. var. cupulatum (Fröhlich 1964)

O. cupulatum Hoffm. ex Brid. var. sardagnanum (Venturi) Venturi (Fröhlich 1964) 
O. diaphanum Schrad. ex Brid. (Fröhlich 1955)

O. pamiricum Plášek \& Sawicki (Plášek et al. 2014)

O. rupestre Schleich. ex Schwägr. var. rupestre (Fröhlich 1964)

O. rupestre Schleich. ex Schwägr. var. sturmii (Hoppe \& Hornsch.) Boulay (new to Afghanistan); KAPISA: Tagab-Alisang, Seken valley, way to Kotal Spe, 2500 m, on rock, 10 September 1974 W. Frey 74-112; TagabAlisang, Kotal Spe, 2150 m, on dry rock, 7 September 1994 W. Frey 74-101

O. tenellum Bruch ex Brid. (Frey \& Kürschner 2009)

O. urnigerum Myrin (Henderson \& Prentice 1963)

\section{Plagiomniaceae}

Plagiomnium rostratum (Schrad.) T.J.Kop. (Kitagawa 1964; cf. Koponen 1982)

\section{Polytrichaceae}

Pogonatum urnigerum (Hedw.) P.Beauv. (new to Afghanistan); BADAKSHAN: Wakhan, Kotal-i Wazit, $37^{\circ} 04^{\circ} \mathrm{N}$ 7252’E, $4600 \mathrm{~m}$, feuchte Stelle, 7. August 1968 S.-W.Breckle 1370a

Polytrichum juniperinum Hedw. (Fröhlich 1955)

\section{Pottiaceae}

Aloina ambigua (Bruch \& Schimp.) Limpr. (Aitchison 1881-1882)

Anoectangium handelii Schiffn. (Fröhlich 1964)

A. thomsonii Mitt. (Herzog 1938)

Barbula bolleana (Müll.Hal.) Broth. (Frey \& Kürschner 2009)

B. unguiculata Hedw. (Fröhlich 1964)

Bryoerythrophyllum recurvirostrum (Hedw.) P.C.Chen [Herzog 1938, Fröhlich 1955, as Didymodon recurvirostris (Hedw.) Jenn. var. obtusifolius J.Fröhl. (holotype)]

B. rubrum (Jur. ex Geheeb) P.C.Chen [Fröhlich 1964, as B. compactum J.Fröhl. (holotype)]

Cinclidotus riparius (Host. ex Brid.) Arn. (Fröhlich 1964, as C. acutifolius Broth.) [Syn. C. acutifolius Broth. (Erdağ \& Kürschner 2011)]

Crossidium squamiferum (Viv.) Jur. var. pottioideum (De Not.) Mönk. (Kürschner 1996)

C. squamiferum (Viv.) Jur. var. squamiferum (Fröhlich 1964)

Didymodon acutus (Brid.) K.Saito (Fröhlich 1964)

D. australasiae (Hook. \& Grev.) R.H.Zander [Fröhlich 1955, as Tortula cabulica J.Fröhl. (holotype) and T. cucullifolia J.Fröhl. (holotype); cf. Jiménez et al. 2005]

D. fallax (Hedw.) R.H.Zander (Fröhlich 1955)

D. icmadophilus (Müll.Hal.) K.Saito (Fröhlich 1964)

D. luridus Hornsch. (new to Afghanistan); BADAKSHAN: Wakhan, Pamir, Issiktal, 5630 m, 10. August 1975 W.Kuschel 1 (GZU), Exploration Pamir 1975; BAGHLAN: Doshi, $1 \mathrm{~km}$ from the village towards Bamyan, 900 m, N-facing slope, 21 May 1972 P.Uotila 17090 (H),KANDAHAR: 55 km W Kandahar, 31 441'N 6453'E, 900 m, Felsrippen, 9 March 1959 S.-W.Breckle; KAPISA: Tagab-Alisang, Kotal Spe, 2000 m, Bewässerungskanal, 7. September 1974 W. Frey 74-72b

D. nicholsonii Culm. [Fröhlich 1964, as Barbula cucullata (holotype)]

D. rigidulus Hedw. (Jiménez 2006)

D. tectorum (Müll.Hal.) K.Saito (new to Afghanistan); BALKH: Mazar-i Sharif, über Aq Kupruk, 700-800 m, K.H.Rechinger 19670b, 19678, 19679, 19680

D. tophaceus (Brid.) Lisa (Fröhlich 1955)

D. vinealis (Brid.) R.H.Zander (Fröhlich 1955)

Eucladium verticillatum (With.) Bruch \& Schimp. (Fröhlich 1955)

Gymnostomum calcareum Nees \& Hornsch. (Henderson \& Prentice 1963)

G. mosis (Lorentz) Jur. \& Milde (Townsend 1966)

G. viridulum Brid. (Whitehouse \& Crundwell 1992)

Hydrogonium gracilentum (Mitt.) P.C.Chen (Fröhlich 1964)

H. patulifolium J.Fröhl. (Fröhlich 1964, holotype)

Hymenostylium recurvirostrum (Hedw.) Dixon var. commutatum (Mitt.) Podp. (Fröhlich 1955)

H. recurvirostrum (Hedw) Dixon var. latifolium (J.E.Zetterst.) Wijk \& Margad. (Fröhlich 1955)

H. recurvirostrum (Hedw.) Dixon var. recurvirostrum [Fröhlich 1964, as H. diversifolium J. Fröhl. (holotype)] Molendoa hornschuchiana (Hook.) Lindb. ex Limpr. [Fröhlich 1964, as Anoectangium pleuroweisioides J. Fröhl. (holotype); Sollman 2006]

Oxystegus tenuirostris (Hook. \& Taylor) A.J.E.Smith (Frey \& Kürschner 2009)

Pseudocrossidium revolutum (Brid.) R.H.Zander (new to Afghanistan); HELMAND: $60 \mathrm{~km}$ N Girishk, 32 ${ }^{\circ} 31^{\prime} \mathrm{N}$ 6446’E, 1400 m, in Felsspalten, 18. März 1969 S.-W.Breckle s.n.; BALKH: Mazar-i Sharif, über Aq Kupruk, 700-800 m, K.H.Rechinger 19671; KANDAHAR: 10 km S Boldak, 1250 m, Felsspalten, 21. März 1969 S.W.Breckle 
Pterygoneurum medium (Salm.) Broth. (Fröhlich 1955)

P. ovatum (Hedw.) Dixon (new to Afghanistan); HERAT: $35 \mathrm{~km} \mathrm{~N}$ Herat, $34^{\circ} 41^{\prime} \mathrm{N} 62^{\circ} 07^{\prime} \mathrm{E}, 1520 \mathrm{~m}$, on soil beneath Iris sp., 15 March 1969 S.-W.Breckle

Stegonia latifolia (Schwägr.) Venturi ex Broth. var. pilifera (Dicks.) Broth. (Fröhlich 1964)

Syntrichia caninervis Mitt. var. caninervis [Fröhlich 1955, 1964, as Tortula pseudodesertorum J.Fröhl. (holotype)]

S. caninervis Mitt. var. gypsophila (J.J.Amann ex G.Roth) Ochyra (Kramer 1978, 1980)

S. caninervis Mitt. var. pseudodesertorum (Vondr.) M.T.Gallego [Fröhlich 1964, as Tortula pseudohandelii

(J.Fröhl.) S.Agnew \& Vondr. (holotype)

S. handelii (Schiffn.) S.Agnew \& Vondr. (new to Afghanistan); HeRAT: 30km W Herat, Ziaret-e Khwaja Koh, c. $34^{\circ} 25^{\prime} \mathrm{N} 61^{\circ} 55^{\prime} \mathrm{E}, 20 \mathrm{~km}$ NW von Herat, $1150 \mathrm{~m}$, unter Brecchien-Felsblöcken, 14 März 1969, S.-W.Breckle 4460a, F1-189; LogAR: bei Sarobi, Cymbopogon-Steppe, März-Juni 1971 O.H.Volk 71253

S. laevipila Brid. [Fröhlich 1955, as Tortula pagorum (Milde) De Not.]

S. princeps (De Not.) Mitt. (Kramer 1980)

S. ruralis (Hedw.) F.Weber \& D.Mohr (Henderson \& Prentice 1963)

S. sinensis (Müll.Hal.) Ochyra (Kilbertus \& Bizot 1971)

S. virescens (De Not.) Ochyra [Kramer 1980, as Tortula ruralis (Hedw.) P.Gaertn., E.Mey. \& Schreb. subsp. ruralis var. substereidosa W.Kramer]

Timmiella anomala (Bruch \& Schimp.) Limpr. (Herzog 1938)

T. barbuloides (Brid.) Mönk. (Herzog 1938, as T. subintegra Dixon, Townsend 1993)

Tortella fragilis (Drumm.) Limpr. (Frey \& Kürschner 2009)

T. inflexa (Bruch) Broth. (new to Afghanistan); HeRAT: $45 \mathrm{~km} \mathrm{~S}$ Herat, 3400’ $\mathrm{N} 62^{\circ} 13^{\prime} \mathrm{E}, 1440 \mathrm{~m}, 14 \mathrm{March}$ 1969 S.-W.Breckle

T. tortuosa (Hedw.) Limpr. (new to Afghanistan); LAGHMAN: Alingar-Seitental, $6 \mathrm{~km}$ W Nangaresh, 34 ${ }^{\circ} 58^{\prime} \mathrm{N}$

70¹9’E, 2400 m, Fels, 9. April 1969 S.-W.Breckle s.n.

Tortula atrovirens (Sm.) Lindb. (Fröhlich 1955)

T. cernua (Huebener) Lindb. (Kilbertus \& Bizot 1971)

T. cuneifolia (Dicks.) Turner (Frey \& Kürschner 2009)

T. inermis (Brid.) Mont. (Henderson \& Prentice 1963)

T laureri (Schultz) Lindb. (Fröhlich 1964)

T mucronifolia Schwägr. (Kramer 1975)

T muralis Hedw. subsp. muralis (Fröhlich 1955)

T. muralis Hedw. subsp. obtusifolia (Schwägr.) Culm. (new to Afghanistan); BADAKSHAN: Wakhan, Khandud-

Tal, 4370 m, Grauschiefer, 15. August 1968 E. Koblmüller

T. subulata Hedw. var. subinermis (Bruch \& Schimp.) Wilson (Herzog 1938)

T. subtranscaspica J.Fröhl. (Fröhlich 1955, holotype)

T. systylia (Schimp.) Lindb. (Fröhlich 1955)

Weissia condensa (Voit) Lindb. (Bizot 1962)

W. controversa Hedw. var. controversa [Herzog 1938, as W. viridula Brid. var. remotidens Herzog (holotype)]

W. controversa Hedw. var. crispata (Nees \& Hornsch.) Nyholm (Frey \& Kürschner 2009)

W. edentula Mitt. (Fröhlich 1955)

W. norkettii Chopra (Fröhlich 1955)

Ptychomitriaceae

Campylostelium saxicola (F.Weber \& D.Mohr) Bruch \& Schimp. (Frey \& Kürschner 2009)

Rhabdoweisiaceae

Dicranoweisia brevifolia Dixon \& Herzog (Herzog 1938, holotype)

Oncophorus virens (Hedw.) Brid. (new to Afghanistan); BADAKSHAN: Darrah-i Parshui, 3700 m, 21. Juli 1965

W. Frey 1624

Splachnobryaceae

Splachnobryum aquaticum Müll.Hal. [Fröhlich 1955, as Hydrogonium heterophyllum (holotype), as S. giganteum Broth., Fröhlich 1964; cf. Arts 2001]

\section{Timmiaceae}

Timmia bavarica Hessl. (Kürschner 1996)

\section{ANTHOCEROTOPHYTA}

Anthoceros spec. (Griffith 1849) 


\section{References}

Bibliography of Afghan bryophytes and general references

Aitchis on J.E.T. (1881-1882): On the flora of the Kuram valley, Afghanistan. - J. Linn. Soc. Bot. 18-19: 1-113, 139-200.

Ando H. (1976): Studies on the genus Hypnum Hedw. (III.). - J. Sci. Hiroshima Univ. Ser. B, Div. 2 (Bot.) 16: 16.

Arts T. (2001): A revision of the Splachnobryaceae (Musci). - Lindbergia 26: 77-96.

Bischler H. (1978): Plagiochasma Lehm. et Lindenb. II. Les taxa Européens et Africains. - Rev. Bryol. Lichén. 44: 223-300.

- (1979): Plagiochasma Lehm. et Lindenb. III. Les taxa d'Asie et d'Océanie. - J. Hattori Bot. Lab. 45: 25-79.

B is chler-Causse H. (1989): Marchantia L. The Asiatic and Oceanic taxa. - Bryoph. Bibl. 38: 1-317.

Bizot M. (1962): Quelques mousses d'Afghanistan. - Rev. Bryol. Lichén. 31: 90.

Blom H.H. (1996): A revision of the Schistidium apocarpum complex in Norway and Sweden. - Bryoph. Bibl. 49: 1-333.

Crundwell A.C. \& Nyholm E. (1977): Dicranella howei Ren. \& Card. and its relationships to D. varia (Hedw.) Schimp. - Lindbergia 4: 35-38.

Erdăg A. \& Kürschner H. (2011): The Cinclidotus P.Beauv / Dialytrichia (Schimp.) Limpr. complex (Bryopsida, Pottiaceae) in Turkey. - Bot. Serbica 35: 13-30.

Frahm J.-P. (1998): Moose als Bioindikatoren. - Quelle \& Meyer Verlag, Wiesbaden.

Frahm J.-P., Stapper N.J. \& Franzen-Reuter I. (2008): Epiphytische Moose als Umweltgütezeiger. Kommission Reinhaltung der Luft im VDI und DIN, KRdL Schriftenreihe 40, Düsseldorf.

Frey W. (1969): Beiträge zur Moosflora Afghanistans I. - Nova Hedwigia 17: 351-357.

- (1972): Beiträge zur Moosflora Afghanistans II. - Die pleurokarpen Laubmoose. - Bryologist 75: 125-135.

- (1974): Die Lebermoose des Iran und Afghanistans. - Bryologist 77: 48-56.

- (1989): The bryophytes of Saudi Arabia and the importance of their conservation. Studies in Arabian bryophytes 9. - In: Abu-Zinada A.H., Goriup P.D. \& Nader I.A. (eds): Wildlife conservation and development in Saudi Arabia. - NCWCD Publ. No. 3: 209-219. Riyadh

Frey W. \& Kürschner H. (1991): Conspectus bryophytorum et arabicorum. An annotated catalogue of the bryophytes of Southwest Asia. - Bryoph. Bibl. 39: 1-181.

- (2009): New records of bryophytes from Afghanistan - with a note on the bryological exploration of the country. - Nova Hedwigia 88: 503-511.

Frey W. \& Stech M. (2009): Marchantiophyta, Bryophyta, Anthocerotophyta. - In: Frey, W. (ed.): Syllabus of Plant Families. Adolf Engler's Syllabus der Pflanzenfamilien, 13 ${ }^{\text {th }}$ ed. - Gebr. Borntraeger Verlagsbuchhandlung, Stuttgart.

Fröhlich J. (1955): Die von Dr. A. Gilli in den Jahren 1949 bis 1951 in Zentral-, Ost- und Nordost-Afghanistan gesammelten Bryophyten. - Mitt. Thüring. Bot. Ges. 1: 59-70.

- (1964): Bryophyten aus Afghanistan und Nordwest-Pakistan. - Ann. Naturhist. Mus. Wien 67: 149-158.

Gallego M.T., Cano M.J., Ros R.M. \& Guerra J. (2002): An overview of the Syntrichia ruralis complex (Pottiaceae: Musci) in the Mediterranean region and neighbouring areas. - Bot. J. Linnean Soc. 138: $209-224$.

Gilli A. (1969): Afghanische Pflanzengesellschaften. - Vegetatio 16: 307-375.

- (1971): Afghanische Pflanzengesellschaften. II. Die mesophilen und hygrophilen Pflanzengesellschaften im sommertrockenen Gebiet. - Vegetatio 23: 199-234.

Greven H.C. (1995): Grimmia Hedw. (Grimmiaceae, Musci) in Europe. - Backhuys Publishers, Leiden.

- (2003): Grimmias of the world. - Buckhuys Publ., Leiden.

Griffith W. (1849): Notulae ad plantas asiaticas, part II. On the higher cryptogamous plants. Calcutta.

Hattori S. (1969): Studies on the Asiatic species of the genus Porella (Hepaticae). II. - J. Hattori Bot. Lab. 32 : 319-358.

- (1978): A note on Asian Porella platyphylla. - Misc. Bryol. Lich. 8: 1-2.

Henderson D.M. \& Prentice H. (1963): Bryophyta. - In: Hedge I.C. \& Wendelbo P. (eds): Studies in the Flora of Afghanistan. 1, pp. 29-31. - Årbok Univ. Bergen Math. - Nat. Ser. 18: 1-56.

Herzog T. (1938): Botanische Ergebnisse der Deutschen Hindukusch-Expedition 1935: Bryophyta. - Feddes Rep. Beih. 108: 3-12.

Jiménez J.A. (2006): Taxonomic revision of the genus Didymodon Hedw. (Pottiaeae, Bryophyta) in Europe, North Africa and Southwest and Central Asia. - J. Hattori Bot. Lab. 100: 211-292.

Jiménez J.A., Ros R.M., Cano M.J. \& Guerra J. (2005): A new evaluation of the genus Trichostomopsis (Pottiaceae, Bryophyta). - Bot. J. Linnean Soc. 147: 117-127.

Kilbertus G. \& Bizot M. (1971): Contribution à la connaissance de la flore des muscinées d'Afghanistan. Bull. Acad. Soc. Lorraine Sci. 10: 67-75. 
Kitagawa N. (1964): Bryophytes in Afghanistan. - In: Kitamura S. (ed.): Results of the Kyoto University Scientific expedition to the Karakoram and Hindu Kush 1955. - 3. Plants of West Pakistan and Afghanistan, pp. 163-166. Kyoto.

Koponen T. (1982): Miscellaneous notes on Mniaceae (Bryophyta). XI. Distribution of Plagiomnium rostratum. - Mem. Soc. Fauna Flora Fennica 58: 17-20.

Kramer W. (1975): Zur Systematik, Ökologie und Geographie der Gattung Tortula (Musci) im Iranischen Hochland. - Unpublished Thesis, Univ. Tübingen.

- (1978): Contribution to the taxonomy and distribution of several taxa of Tortula Hedw. sect. Rurales De Not. (Musci) with bistratose lamina. - Bryologist 81: 378-385.

- (1980): Tortula Hedw. sect. Rurales De Not. (Pottiaceae, Musci) in der östlichen Holarktis. - Bryoph. Bibl. 21: 1-165.

Kürschner H. (1996): Towards a bryophyte flora of the Near and Middle East. - New records from Iran, Jordan, Kuwait, Lebanon, Oman, Saudi Arabia, Syria and Turkey. - Nova Hedwigia 63: 261-271.

- (2001): Towards a bryophyte flora of the Near and Middle East. 3. An artificial key to the Anthocerorotophytina and Hepaticophytina of the Near and Middle East. - Nova Hedwigia 72: 161-200.

- (2006): A key to the pleurocarpous mosses (Bryophytina p.p.) of the Near and Middle East. Towards a bryophyte flora of the Near and Middle East, 5. - Nova Hedwigia 83: 353-386.

- (2007): A key to the Pottiaceae (Bryopsida - Bryophytina) of the Near and Middle East. Towards a bryophyte flora of the Near and Middle East, 6. - Nova Hedwigia 84: 21-50.

- (2008): A key to the pleurocarpous mosses (Bryophytina p.p., excl. Pottiaceae) of the Near and Middle East. Towards a bryophyte flora of the Near and Middle East, 7. - Nova Hedwigia 86: 43-103.

Kürschner H. \& Frey W. (2006): Tortula grandiretis Broth. (Pottiaceae), an Irano-Turanian moss new to Iraq and further novelties from the Near and Middle East. Towards a bryophyte flora of the Near and Middle East, 4. - Nova Hedwigia 83: 143-149.

- (2011): Liverworts, Mosses and Hornworts of Southwest Asia. Marchantiophyta, Bryophyta, Anthocerotophyta. - Nova Hedw. Beih. 139: 1-240.

Kürschner H., Lüth M. \& Frey W. (2019): Field Guide Afghanistan. Liverworts and Mosses. - Breckle, S.-W., Hedge, I.C. \& Rafiqpoor, M.D. (eds.). - United Nations Office Geneva (in press).

Lewinsky J. (1992): The genus Orthotrichum Hedw. (Orthotrichaceae, Musci) in Southeast Asia. A taxonomic revision. - J. Hattori Bot. Lab. 72: 1-88.

Maier E. (2002): Grimmia dissimulata E. Maier sp. nova, and the taxonomic position of Grimmia trichophylla var. meridionalis Müll. Hal. (Musci, Grimmiaceae). - Candollea 56: 281-300.

- (2010): The genus Grimmia Hedw. (Grimmiaceae, Bryophyta) - A morphological-anatomical study. - Boissiera 63: 5-377.

Müller K. (1954-1957): Die Lebermoose Europas. - In: Rabenhorst L., Kryptogamenflora von Deutschland, Österreich und der Schweiz. - Leipzig.

Muñoz J. (1998): A taxonomic revision of Grimmia Subgenus Orthogrimmia (Musci, Grimmiaceae). - Ann. Missouri Bot. Gard. 85: 367-403.

- (1999): A revision of Grimmia (Musci, Grimmiaceae) in the Americas. - Ann. Missouri Bot. Gard. 86: 118-191.

Muñoz J. \& Pando F. (2000): A world synopsis of the genus Grimmia. - Monogr. Syst. Bot. Missouri Bot. Gard. 83: $1-133$.

Plášek V., Sawicki J. \& Číhal L. (2014): Orthotrichum pamiricum (Bryophyta), a new epiphytic species from Pamir Mountains in Central Asia. - Turk. J. Bot. 38: 754-762.

Ros M.R., Mazimpaka V., Abou-Salama U., Aleffi M., Blockeel T.L., Brugués M., Cano M.J., Cros R.M., Dia M.G., Dirkse G.M., et al. (2007): Hepatics and Anthocerotae of the Mediterranean, an annotated checklist. - Cryptogamie, Bryologie 28: 351-437.

Ros R.M., Mazimpaka V., Abou-Salama U., Aleffi M., Blockeel T.L., Brugués M., Cros R.M., Dia M.G., Dirkse G.M., Draper I., et al. (2013): Mosses of the Mediterranean, an annotated checklist. Cryptogamie, Bryologie 34: 99-283.

Salmon E.S. (1899): A new moss from Afghanistan. - J. Bot. 37: 241-242.

Sollman P. (2005): Studies on some Asian mosses of the Pottiales, mainly from the Himalayas. - Trop. Bryol. 26: $81-88$

- (2006): Studies on several Asian Pottiaceous mosses, mainly from the Himalayas. - Trop. Bryol. 28 : 5-7.

Townsend C.C. (1966): Mosses from Iran and Afghanistan. - Transact. Brit. Bryol. Soc. 5: 131-135.

- (1993): New records and a bibliography of the mosses of Pakistan. - J. Bryol. 17: 671-678.

Vitt D.H. (1972): A monograph of the genus Drummondia. - Can. J. Bot. 50: 1191-1208.

Whitehouse H.L.K. \& Crundwell A.C. (1992): Gymnostomum calcareum Nees \& Hornsch. and G. viridulum Brid. in Europe, North Africa and the Middle East. - Bull. Brit. Bryol. Soc. 59: 35-50.

Zander R.H. (1993): Genera of the Pottiaceae: mosses of harsh environments. - Bull. Buffalo Soc. Nat. Sci. 32 : 1-378. 
Authors' addresses: Harald Kürschner, Freie Universität Berlin, Institut für Biologie, Altensteinstr. 6, 14195 Berlin, Germany.

E-mail: kuersch@zedat.fu-berlin.de

Wolfgang Frey, Ortlerweg 39d, 12207 Berlin, Germany.

E-mail: wgfrey@gmx.de 